\title{
Remote Attestation with Domain-Based Integrity Model and Policy Analysis
}

\author{
Wenjuan Xu, Xinwen Zhang, Member, IEEE, Hongxin Hu, Student Member, IEEE, \\ Gail-Joon Ahn, Senior Member, IEEE, and Jean-Pierre Seifert, Member, IEEE
}

\begin{abstract}
We propose and implement an innovative remote attestation framework called DR@FT for efficiently measuring a target system based on an information flow-based integrity model. With this model, the high integrity processes of a system are first measured and verified, and these processes are then protected from accesses initiated by low integrity processes. Toward dynamic systems with frequently changed system states, our framework verifies the latest state changes of a target system instead of considering the entire system information. Our attestation evaluation adopts a graph-based method to represent integrity violations, and the graph-based policy analysis is further augmented with a ranked violation graph to support high semantic reasoning of attestation results. As a result, DR@FT provides efficient and effective attestation of a system's integrity status, and offers intuitive reasoning of attestation results for security administrators. Our experimental results demonstrate the feasibility and practicality of DR@FT.
\end{abstract}

Index Terms-Remote attestation, platform integrity, security policy, policy analysis.

\section{INTRODUCTION}

$I^{N}$ $\mathrm{N}$ distributed computing environments, it is crucial to measure whether a remote party runs buggy, malicious application codes or is improperly configured. Remote attestation techniques have been proposed for this purpose by analyzing the integrity of a remote system to determine its trustworthiness. Typical attestation mechanisms are designed based on the following steps. First, an attestation requester (attester) sends a challenge to a target system (attestee), which responds with the evidence of the integrity of its hardware and software components. Second, the attester derives runtime properties of the attestee and determines the trustworthiness of the attestee. Finally and optionally, the attester returns an attestation result, such as integrity status, to the attestee.

Various attestation approaches and techniques have been proposed. Trusted Computing Group (TCG) [1] introduces trusted platform module (TPM) which enables integrity measurements of a remote system. In addition, integrity measurement mechanisms facilitating the capabilities of TPM at application level have been proposed. For instance, Integrity Measurement Architecture (IMA) [2] is an implementation of TCG approach to provide verifiable evidence

- W. Xu is with the Department of Computer Science and Information Technologies, Frostburg State University, 101 Braddock Road Frostburg, MD 21532. E-mail: wxu@frostburg.edu.

- X. Zhang is with Huawei Research Center, 1711 Winston Street, San Jose, CA 95131. E-mail: xinwen.zhang@huawei.com.

- H. Hu and G.-J. Ahn are with Ira A. Fulton School of Engineering, Arizona State University, PO Box 878809, Tempe, AZ 85287. E-mail: $\{h x h u, g a h n\} @ a s u . e d u$.

- J.-P. Seifert is with Deutsche Telekom Laboratories and Technical University of Berlin, Fakultat IV, Sekr. TEL11, Ernst-Reuter-Platz 7, Berlin 10587, Germany. E-mail: jean-pierre.seifert@telekom.de.

Manuscript received 27 Dec. 2010; revised 31 Aug. 2011; accepted 4 Nov. 2011; published online 7 Dec. 2011.

Recommended for acceptance by X. Wang.

For information on obtaining reprints of this article, please send e-mail to: tdsc@computer.org, and reference IEEECS Log Number TDSC-2010-12-0238. Digital Object Identifier no. 10.1109/TDSC.2011.61. with respect to the current runtime state of a measured system. Moreover, several attestation methods have been introduced to address privacy properties [3], system behaviors [4], and information flow model [5]. However, existing approaches have several limitations that obstruct their wide deployment in real systems. First of all, TCG-based mechanisms are inefficient, since IMA need to verify all software components of a running system. Furthermore, without a build-in runtime integrity model, an attestation verification procedure cannot evaluate the integrity status with respect to the trust level of the system. The problems become worse when the attestation target is a dynamic system with continuously changing states due to some system-centric events, such as security policy updates and software package installations. Last but not least, existing attestation mechanisms cannot provide an effective and intuitive way to represent attestation results and reflect such results in integrity violation resolution.

Toward a systematic attestation solution, we propose an efficient remote attestation framework, called dynamic remote attestation framework and tactics (DR@FT), to address aforementioned issues. Our framework is based on a domain-based integrity model to describe the integrity status of a system with information flow control. With this property, the high integrity processes of a system are first measured and verified, and these processes are then protected from accesses initiated by low integrity processes during runtime. In other words, the protection of high integrity process is verified by analyzing security policies and ensuring that the policies are correctly enforced. Having this principle in place, DR@FT enables us to verify whether certain applications (domains) in the attestee satisfy integrity requirements without verifying all components of the system. To accommodate the dynamic nature of a system, DR@FT only verifies the latest changes in a system state, instead of considering the entire system information for each attestation inquiry. Through these two 
tactics, our framework is able to achieve an efficient attestation to the target system. Also, DR@FT adopts a graph-based information flow analysis mechanism to examine security policy violations based on our integrity model, which helps cognitively identify suspicious information flows in the attestee. To further improve the efficiency of security violation resolution, we propose a ranking scheme for prioritizing policy violations, which provides a method for describing the trustworthiness of different system states with risk levels.

Overall, the contribution of this paper is three-fold:

- $\quad$ First, we propose a domain-based integrity model to describe the integrity and thus trustworthiness of a general computing system. Built on information flow control, the model is leveraged to identify the trusted computing base (TCB) of a system (system TCB) and trusted subjects and objects of an application domain (domain TCB). For integrity protection purpose, we define information flow control rules to protect the integrity of system TCB and domain TCBs. Our integrity model and information flow control rules serve as a theoretical foundation for attesting the trustworthiness of a system.

- We then introduce DR@FT along with its architecture, protocols, and algorithms for measuring TCB subjects and objects, reporting measurements to attester, and evaluating integrity status of the attestee based on our proposed integrity model. We present the tactics to achieve efficient attestation processing with the measurements of TCB subjects at attestee side and accelerated verification processing based on policy update at attester side.

- To assist integrity verification, we also develop a graph-based policy analysis tool, which automatically checks possible integrity flow violations based on our integrity model. Based on a set of information flow control rules, our graph-based analysis tool further provides intuitiveness on how to resolve violations, which can be sent as the attestation result to the attestee. To measure the risks of variant information flow violations, we further propose a ranked policy violation graph, which can be used to guide the system administrator for effectively resolving identified violations.

This paper is organized as follows: Section 2 briefly overviews existing system integrity models, policy analysis, and attestation mechanisms. Section 3 describes our domain-based integrity model which provides a theoretical foundation of DR@FT. Section 4 presents design requirements and attestation procedures of DR@FT. We then illustrate our graph-based policy analysis tool in Section 5, followed by an introduction of our ranked policy violation graph and its application. We elaborate the implementation details and evaluation results in Section 7. Section 8 concludes this paper.

\section{Background and Related Work}

\subsection{Integrity Models}

To describe and maintain the integrity status of a system, there exist various information flow-based integrity models such as Biba [6], LOMAC [7], Clark-Wilson [8], and CW-Lite [9]. Biba integrity property is fulfilled if a high-integrity process cannot read/execute a lower integrity object, nor obtains lower integrity data in any other manner. LOMAC allows high-integrity processes to read lower integrity data, while downgrades the process's integrity level to the lowest integrity level that has ever been activated. Clark-Wilson provides a different view of dependencies, which states information flow from lowintegrity objects to high-integrity objects through a specific program called transaction procedures (TP). Later, the concept of TP is evolved as a filter in the CW-Lite model. The filter can be a firewall, an authentication process, or a program interface for downgrading or upgrading the privileges of a process.

With existing integrity models, there is a gap between concrete measurements of a system's components and the verification of its integrity status. We believe an applicationoriented and domain-centric approach accommodates the requirements of attestation evaluation better than directly adopting an abstract model. For example, in a Linux system, a subject in traditional integrity models corresponds to a set of processes, which belong to a single application domain. A more concrete instance is that an Apache domain may include various process types such as httpd_t, http_sysadm_ devpts_t, and httpd_prewikka_script_t, and all of these types may have information flows among them, which should be regarded as a single-integrity level. Also, sensitive objects in a domain should share the same integrity protection of its subjects. In this paper, we propose a domain-based isolation model to capture the essence of application- or domain-centric integrity requirements.

\subsection{Policy Analysis}

Jaeger et al. [10] and Shankar et al. [11] use a tool called Gokyo for checking the integrity of a proposed TCB for SELinux [12]. Also, they attempt to implement their ideas in an automatic manner. Gokyo mainly identifies a common TCB in SELinux but a typical system may have multiple applications and services with variant trust relationships. Achieving the integrity assurance for these applications and services was not addressed in Gokyo. Policy analysis on SELinux is further proposed in [13] toward MLS security properties.

Several query-based policy analysis tools have been developed. APOL [14] is a tool developed by Tresys Technology to analyze SELinux policies by computing an information flow relation and the transitive closure of the relation. Security Enhanced Linux Analysis Tool (SLAT) [15] defines an information flow model and policies are analyzed based on this model. Policy Analysis using Logic Programming (PAL) [16] uses SLAT information flow model to implement a framework for analyzing SELinux policies. All these tools try to provide a way for querying policies. However, they only display policies and policy query results in a text-based fashion, which is difficult to understand for policy developers or security administrators.

To overcome these limitations, we have developed a graph-based methodology for identifying and expressing interested information flows in SELinux policies [17]. We have also proposed a policy analysis mechanism using Petri Nets presented in [18]. However, these tools and mechanisms 
are limited in identifying system TCB and domain TCBs, but do not have the capability of identifying and ranking integrity violations thus providing evidential support for attestation results.

\subsection{Remote Attestation}

TCG specifications [1] define mechanisms for a TPMenabled platform to report its current hardware and software configuration status to a remote challenger. A TCG attestation process is composed of two steps: 1) an attestee platform measures hardware and software components starting from BIOS block and generates a hash value. The hash value is then stored into a TPM Platform Configuration Register (PCR). Recursively, it measures BIOS loader and operating system (OS) in the same way and stores them into TPM PCRs; 2) an attester obtains the attestee's digital certificate with an attestation identity key (AIK), AIK-signed PCR values, and a measurement log file from the attestee which is used to reconstruct the attestee platform configuration, and verifies whether this configuration is acceptable. From these steps, we notice that TCG measurement process is composed of a set of sequential steps including the bootstrap loader. Thus, TCG does not provide effective mechanisms for measuring a system's integrity beyond the system boot, especially considering the randomness of executable contents loaded by a running OS.

IBM IMA [2] extends TCG's measurement scope to application level. A measurement list $M$ is stored in OS kernel and composed of $m_{0} \ldots m_{i}$ corresponding to loaded executable application codes. For each loaded $m_{i}$, an aggregated hash $\mathrm{H}_{i}$ is generated and loaded into TPM PCR, where $\mathrm{H}_{0}=\mathrm{H}\left(m_{0}\right)$ and $\mathrm{H}_{i}=\mathrm{H}\left(\mathrm{H}_{i-1} \| \mathrm{H}\left(m_{i}\right)\right)$. Upon receiving the measurements and TPM-signed hash value, the attester proves the authentication of measurements by verifying the hash value, which helps to determine the integrity level of the platform. However, IMA requires to verify the entire components of the attestee platform while the attestee may only demand the verification of certain applications. Also, the integrity status of a system is validated by testing each measurement entry independently, and it is impractical to disregard newly installed untrusted applications or data from the untrusted network.

PRIMA [5] is an attestation mechanism based on IMA and CW-Lite integrity model [9], and attempts to improve the efficiency of attestation by verifying only codes, data, and information flows related to trusted subjects. On one hand, PRIMA needs to be extended to capture the dynamic nature of system states such as software and policy updates, which could be an obstacle for maintaining the efficiency. On the other hand, PRIMA only represents an attestation result with binary decisions (either trust or distrust) and does not give semantic information about how much the attestee platform can be trusted.

Property-based attestation [3] is an effort to protect the privacy of a platform by collectively mapping related system configurations to attestation properties. For example, "SELinux-enabled" is a property which can be mapped to a system configuration indicating that the system is protected with an SELinux policy. That is, this approach prevents the configurations of a platform from being disclosed to a challenger. However, due to the immense configurations of the hardware and software of the platform, mapping all system configurations to properties is infeasible and impractical.

Semantic remote attestation [4] is proposed with a trusted virtual machine running on a platform. Through monitoring the policy attached to a running software, its behavior is verified. However, this approach does not define the correct behavior of a security policy with respect to integrity, which leads the attestation to be intractable. Behavior-based attestation [19] attempts to attest system behaviors based on an application level policy model. Such a model-based approach may not comprehensively realize the complex behaviors of dynamic systems.

\section{Domain-Based INTEgRITY MOdel}

According to TCG and IMA, the trustworthiness of a system is described with the measured integrity values (hash values) of loaded software components. However, the measurement efficiency and attestation effectiveness are major problems of these approaches since 1) too many components have to be measured and tracked, 2) too many known-good hash values are required from different software vendors or authorities, and 3) runtime integrity cannot be guaranteed and verified. Fundamentally, in order to trust a single application of a system, every software component in the system has to be trusted; otherwise the attestation result should be negative. In our work, we believe that the trustworthiness of a system is tightly related to the integrity status, which is, in turn, described by a set of integrity rules that are enforced by the system. If any of the rules is violated, it should be detected. Hence, so as to trust a single application domain, we just need to ensure the TCB-including reference monitor and integrity rules protecting the target application domain-is not altered.

Based on this anatomy, we introduce domain-based isolation principles for integrity protection, which are the criteria to describe the integrity status of a system as well as its trustworthiness. We first propose general methodologies to identify high-integrity processes, which include system $T C B$ and domain $T C B$. We then specify security rules for protecting these high-integrity processes.

\subsection{System ТСВ}

The concept of system TCB $\left(T C B_{s}\right)$ is similar to that of traditional TCB [20], which can be identified along with the subjects functioning as the reference monitor of a system [21]. Applying this concept to SELinux [22], for example, subjects functioning as the reference monitor such as checkpolicy and loading policy belong to system TCB. Also, subjects used to support the reference monitor such as kernel and initial should also be included into system TCB. With this approach, an initial $T C B_{s}$ can be identified, and other subjects such as $1 \mathrm{vm}$ and restorecon can be added into $T C B_{s}$ based on their relationships with the initial $T C B_{s}$. Other optional methods for identifying $T C B_{s}$ are proposed in [10]. Considering the similarity of operating systems and configurations, we expect that the results would be similar. Furthermore, for 
the attestation purpose, $T C B_{s}$ also includes integrity measurement and reporting components, such as kernel level integrity measurement agent [23] and attestation request handling agent.

\subsection{Domain TCB}

In practice, other than $T C B_{s}$, an application or user-space service can also affect the integrity and the behavior of a system. An existing argument [20] clearly states the situation: "A network server process under a UNIX-like operating system might fall victim to a security breach and compromise an important part of the system's security, yet is not part of the operating system's TCB." Accordingly, a comprehensive mechanism of policy analysis for TCB identification and integrity violation detection is desired. Hence, we introduce a concept called information domain TCB (or simply domain TCB, TCB $B_{d}$ ). Let $d$ be an information domain functioning as a certain application or service through a set of related subjects and objects. A domain $d$ 's TCB or $T C B_{d}$ is composed of a set of subjects and objects in information domain $d$ which have the same level of integrity. By the same level of integrity, we mean that, if information can flow to some subjects or objects of a domain, it can flow to all others in the same domain with legitimate operations of the application. That is, they need the same level of integrity protection. Prior to the identification of $T C B_{d}$, we first identify the information domain $d$ based on its main functions and relevant information flow associated with these functions. For example, a running web server domain consists of many subjects-such as httpd process, plugins, and tools, and other objects-such as data files, config files, and logs. ${ }^{1}$

The integrity of an object is determined by the integrity of subjects that have operations on this object. All objects dependent on $T C B_{d}$ subjects are classified as TCB protected objects or resources. Thus, we need to identify all $T C B_{d}$ subjects from an information domain and verify the assurance of their integrity. To ease this task, a minimum $T C B_{d}$ is first discovered. In the situation that the minimum $T C B_{d}$ subjects have dependency relationships with other subjects, these subjects should be added to domain TCB, or the dependencies should be removed. Based on these principles, we first identify initial $T C B_{d}$ subjects which are predominant subjects for the information domain $d$. We further discover other $T C B_{d}$ subjects considering subject dependency relationships with the initial $T C B_{d}$ through information flow transitions, which means subjects that can only flow to and from initial $T C B_{d}$ subjects should be included into $T C B_{d}$. For instance, for a web server domain, httpd is the subject that initiates other web server related processes. Hence, httpd is the predominant subject and belongs to $T C B_{d}$. Based on all possible information flows to httpd, we then identify other subjects such as httpdsuexec in $T C B_{d}$.

\subsection{Integrity Protection with Domain Isolation}

To protect the identified $T C B_{s}$ and $T C B_{d}$, we develop principles similar to those in Clark-Wilson [8]. ClarkWilson leverages TP to allow information flow from lowintegrity processes to high-integrity processes. To support

1. Section 7 shows the Apache domain TCB in our evaluation system.
TP, we adopt the concept of filters. Filters can be processes or interfaces [24] that normally are distinct input information channels and are created by a particular operation such as open ( ), accept (), or other calls that enable data input. For example, su process allows a low-integrity process (e.g., staff) being changed to be a high-integrity process (e.g., root) by executing passwd process, thus passwd can be regarded as a filter for processes run by root privilege. Also, high-integrity process (e.g., httpd administration) can accept low-integrity information (e.g., network data) through the secure channel such as sshd. Consequently, sshd can be treated as a filter for higher privilege processes. In general, filters include any available sanitization process and TP in traditional integrity model. Since it is usually very difficult to build and completely verify a sanitization process, we believe it is more reasonable to assume that the system is in higher trusted state when any low-to-high information flow is explicitly authorized by a user.

With the identifications of $T C B_{s}, T C B_{d}$, and filters for information domain $d$, all the other subjects in a system are categorized as NON-TCB. Our domain-based isolation is defined as follows:

Definition 1. Domain-based isolation is satisfied for an information domain $d$ if

- information flows are from $T C B_{d}$; or

- information flows are from $T C B_{s}$ to either $T C B_{d}$ or $T C B_{d}$ protected resources; or

- information flows are from NON-TCB to either $T C B_{d}$ or $T C B_{d}$ protected resources via filter(s).

Deductively, we articulate the rule for integrity evaluation based on domain-based isolation.

Rule 1. If there is information flow from NON-TCB to TCB without passing through any filter, there is an integrity violation related to $T C B_{d}$ protected resources.

Rule 2. Or if there is information flow from NON-TCB or TCB to $T C B_{s}$ without passing through any filter, there is an integrity violation related to $T C B_{s}$ protected resources.

\section{DR@FT: Design and Procedures}

DR@FT consists of three main parties: an attestee (the target platform), an attester (attestation challenger), and a trusted authority as shown in Fig. 1. The attestee is required to provide its system state information to the attester for verification. Here, we assume that the attestee is initially in a trusted system state and the system state is changed to a new state after certain system behaviors.

A reporting daemon on the attestee gets the measured new state information (step 1) with IMA and generates the policy updates (step 2). This daemon then gets AIK-signed PCR value(s) and sends to the attester. After the attester receives and authenticates the information, with the attestee's AIK public key certificate from the trusted authority, it verifies the attestee's integrity through codes and data verification (step 3), reporting process authentication (step 4) and policy analysis (step 5). 


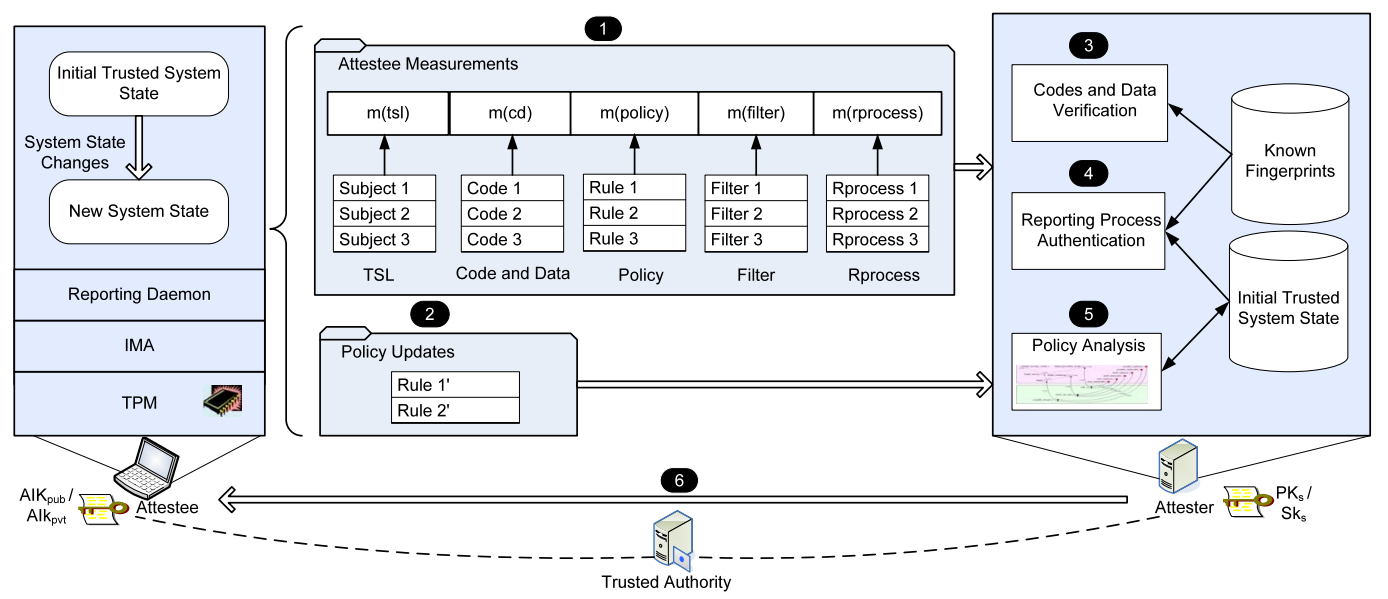

Fig. 1. Overview of DR@FT.

\subsection{System State and Trust Requirement}

For attestation purpose, a system state is captured as a snapshot of the attestee system at a particular moment, where the factors characterizing the state can influence the system integrity on any future moment of the attestee system. Based on our domain-based integrity model, the attestee system integrity can be represented via information flows, which are characterized by the trusted subject list, filters, policies, and the trustworthiness of $T C B_{s}$. Based on these properties, we define the system state of the attestee as follows:

Definition 2. A system protection state (or simply system state) at the time period $i$ is a tuple $T_{i}=\left\{T S L_{i}, C D_{i}\right.$, Policy $_{i}$, Filter $_{i}$, RProcess $\left._{i}\right\}$, where

- $\quad T S L_{i}=\left\{s_{0}, s_{1} \ldots s_{n}\right\}$ represents a set of high-integrity processes which corresponds to a set of subjects $s_{0}, s_{1} \ldots s_{n}$ in $T C B_{s}$ and $T C B_{d}$;

- $C D_{i}=\left\{c d\left(s_{0}\right), \operatorname{cd}\left(s_{1}\right) \ldots c d\left(s_{n}\right)\right\}$ is a set of codes and data for loading a subject $s_{j} \in T S L_{i}$;

- Policy $_{i}$ is the security policy currently configured on the attestee;

- Filter $_{i}$ is a set of processes defined to allow information flow from low-integrity processes to high-integrity processes; and

- RProcess $s_{i}$ represents a list of processes that measure, monitor, and report the current $T S L_{i}, C D_{i}$, Filter $_{i}$, and Policy information. IMA agent and the attestation reporting daemon are the examples of the RProcess . $_{\text {. }}$

According to this definition, a system state does not include a particular application's running state such as its memory page and CPU context (stacks and registers). It only represents the security configuration or policy of an attestee system. A system state transition indicates one or more changes in $T S L_{i}, C D_{i}$, Policy $_{i}$, Filter $_{i}$, or RProcess . $_{i}$. A system state $T_{i}$ is trusted if $T S L_{i}$ belongs to $T C B_{s} \cup T C B_{d}$, $C D_{i}$ does not contain untrusted codes and data, Policy satisfies domain-based isolation, Filter $_{i}$ belongs to defined filters in domain-based isolation, and RProcess $s_{i}$ codes and data do not contain malicious codes and data and these RProcess $_{i}$ processes are integrity protected from the untrusted processes via Policy . $_{\text {. }}$
As mentioned earlier, we assume that there exists an initial trusted system state $T_{0}$. Through changing the variables in $T_{0}$, the system transits to other states such as $T_{1}, \ldots, T_{i}$. The attestation in DR@FT is to verify whether or not $T_{i}$ is trusted.

\subsection{Attestation Procedures}

\subsubsection{Integrity Measurements}

The measurement at the attestee side has two different forms, depending on how much the attestee system changes. Specifically, in case any subject in $T C B_{s}$ is updated, the attestee must be fully remeasured from the system reboot and the attester needs to attest it completely, since this subject may affect the integrity of subjects in RProcess of the system such as the measurement agent and reporting daemon. After the reboot and all $T C B_{s}$ subjects are remeasured, a trusted initial system state $T_{0}$ is built. To perform the remeasurement, the attestee measures a state $T_{i}$ and generates the measurement list $M_{i}$ which is added by trusted subject list $\left(T S L_{i}\right)$ measurement, codes and data $\left(C D_{i}\right)$ measurement, policy (Policy $)_{i}$ measurement, filter $\left(\right.$ Filter $\left._{i}\right)$ measurement, and attestation process (RProcess $\left.s_{i}\right)$ measurement, as explained as follows:

- Trusted subject list $\left(T S L_{i}\right)$ measurement: with TPM and measurement mechanisms such as IMA, the trusted subject list $T S L_{i}$ is measured with a result $m_{t s l}$, which is added to a measurement list by $M_{i}=M_{i} \| m_{t s l} . \mathcal{H}\left(M_{i}\right)$ is extended to a particular PCR of the TPM, where $\mathcal{H}$ is a hash function such as SHA1.

- Codes and data $\left(C D_{i}\right)$ measurement: for every subject $s_{j}$ in $T S L_{i}$, its codes and data $c d\left(s_{j}\right)$ are measured. To specify the mapping relationship, the measurement $m_{c d s j}$ consists of the information of $c d\left(s_{j}\right)$ and $s_{j}$. After $m_{c d s j}$ is generated, it is added to the measurement list by $M_{i}=M_{i} \| m_{c d s j}$ and its hash value is extended to PCR.

- Policy $\left(\right.$ Policy $\left._{i}\right)$ measurement: corresponding to Policy $_{i}$, the attestee generates the measurement $m_{p}$, which is added to the measurement list with $M_{i}=M_{i} \| m_{p}$, and corresponding PCR is extended with its hash value. 
- Filter $\left(\right.$ Filter $\left._{i}\right)$ measurement: the codes and data of Filter $_{i}$ are measured as $m_{f}$, which is extended to the measurement list $M_{i}=M_{i} \| m_{f}$, and the PCR is extended.

- Attestation Process (RProcess $s_{i}$ ) measurement: the codes and data of AProcess $s_{i}$ are measured as $m_{r}$, which is added to the measurement list $M_{i}=M_{i} \| m_{r}$, and the PCR is also extended.

In another case, where there is no $T C B_{s}$ subject

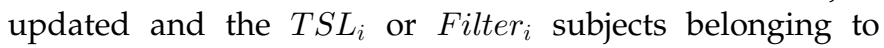
$T C B_{d}$ are updated, the attestee only needs to measure the updated codes and data loaded by the changed TSL or filter subjects, and generates a measurement list $M_{i}$. The generation of this measurement list is realized through the runtime measurement supported by the underlying measurement agent.

To support both types of measurements, we develop an attestation reporting daemon which monitors the runtime measurements of the attestee. In case the runtime measurements for $T C B_{s}$ are changed, the attestee is required to be rebooted and fully measured with IMA. The measurements values are then sent to the attester by the daemon. On the other side, the changed measurement value is measured by IMA and captured with the reporting daemon only if the measurement for $T C B_{d}$ is changed. Obviously, this daemon should be trusted and is included in $T C B_{s}$. That is, its codes and data are required to be protected with integrity policy and corresponding hash values are required to be stored at the attester side.

\subsubsection{Policy Updates}

To analyze if the current state of the attestee satisfies domain-based integrity property, the attester requires information about the current security policy loaded at the attestee side. Due to the large volume of policy rules in a security policy, sending all policy rules in each attestation and verifying all of them by the attester may cause the performance overhead. Hence, in DR@FT, the attestee only generates policy updates from the latest attested trusted state and sends them to the attester for the attestation of such updates.

To support this mechanism, we have the attestation reporting daemon to monitor any policy update on attestee system and generate a list of updated policy rules. The algorithm of this operation is shown in Algorithm 1. Upon the system policy Policy in $_{1}$ is changed on state $T_{i-1}$, the daemon process reads the policy rules in the stored security policy Policy $_{0}$ of the trusted system state $T_{0}$ and the current

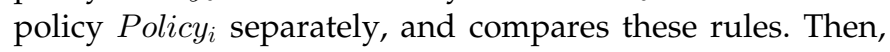
the daemon finds the added, changed, and deleted rules through functions added, changed, deleted, respectively, and saves them into the policy update file. Note that the policy update comparison is performed between the current updated policy and the stored trusted security policy Policy $_{0}$ or previously attested and trusted Policy $y_{i-1}$. The complexity of this policy update algorithm is $O(n r)$, where $n r$ represents the number of the policy rules in the new policy file Policy.
Algorithm 1. Generating Policy Updates

Input: Currently configured policy file $P_{i}$, stored trusted policy file $P_{0}$, currently running updates thread $U_{s}$

Output: Policy updates file $P_{\text {updates }}$

state $:=$ threadMonitor $\left(U_{s}, P_{i}\right)$

if $i s$ Changed(state) then

$$
\begin{aligned}
& \text { fileFlow }:=\text { Initialize }\left(P_{\text {updates }}\right) \\
& R_{\text {list }}:=\operatorname{readPolicy}\left(P_{i}\right) \\
& \text { foreach } r_{i} \in R_{\text {list }} \text { do } \\
& \text { compareResult }:=\left(r_{i}, P_{0}\right) \\
& \text { if compareResult }==1 \text { then } \\
& \text { | added(fileFlow, } \left.r_{i}\right) \\
& \text { else } \\
& \text { if compareResult }==2 \text { then } \\
& \text { changed (fileFlow, } \left.P_{0}, r_{i}\right) \\
& \text { else } \\
& \operatorname{markPolicy}\left(P_{0}, r_{i}\right) \\
& \operatorname{markPolicy}\left(P_{0}, r_{i}\right) \\
& R_{\text {list }}:=\operatorname{readPolicy}\left(P_{0}\right) \\
& \text { foreach } r_{i} \in R_{\text {list }} \text { do } \\
& \text { if } i \text { snotMarked }\left(P_{0}, r_{i}\right) \text { then } \\
& \left\llcorner\text { deleted }\left(\text { fileFlow, } r_{i}\right)\right.
\end{aligned}
$$

\subsubsection{Codes and Data Verification}

With received measurement list $M_{i}$ and AIK-signed PCRs, the attester first verifies the measurement integrity by reconstructing the hash values and compares with PCR values. After this is passed, the attester performs the analyses. Specifically, it obtains the hash values of $C D_{i}$ and checks if they corresponds to known-good fingerprints. Also, the attester needs to assure that the $T S L_{i}$ belongs to $T C B_{s}$ and $T C B_{d}$. In addition, the attester also gets the hash value of Filter $_{i}$ and ensures that they belong to the filter list defined on the attester side. In case this step succeeds, the attester has the assurance that target processes on attestee side are proved without containing any untrusted code or data, and the attester can proceed to next steps. Otherwise, the attester sends a proper attestation result denoting this situation.

\subsubsection{Reporting Process Authentication}

To prove that the received measurements and updated policy rules are from the attestee, the attester authenticates them by verifying that all the measurements, updates, and integrity measurement agent processes in the attestee are protected. That is, the RProcess $s_{i}$ does not contain any untrusted codes or data and its measurements correspond to PCRs in the attester. Also, there is no integrity violated information flow to these processes from subjects of $T S L_{i}$, according to the domain isolation rules. Note that these components can also be updated, but after any update of these components, the system should be fully remeasured and attested from the bootstrap to rebuild a trusted initial system state $T_{0}$.

\subsubsection{Policy Analysis by Attester}

DR@FT analyzes policy using a graph-based analysis method. In this method, a policy file is first visualized 
with a graph. The policy graph is then analyzed against a predefined security model, such as our domain-based isolation model, and a policy violation graph is generated. The main goal of this intuitive approach is to give semantic information of attestation result to the attestee, such that system administrators can easily recognize any violated configuration.

Note that verifying all of the security policy rules in each attestation request decrease the efficiency, as loading policy graph and checking all of the policy rules are costly. Thus, we need to develop an efficient way for analyzing the attestee policy. In our method, the attester stores the policy of initial trusted system state $T_{0}$ or the latest trusted system state $T_{i}$, and loads its corresponding policy graph, which does not have any policy violation. Upon receiving the updated information from the attestee, the attester just needs to analyze these updates to examine if there is new information flow violating integrity requirements. The details of graph-based policy analysis are explained in Section 5 .

\subsubsection{Attestation Result Sending to Attester}

In case the attestation is successful, a new trusted system state is built and the corresponding information is stored at the attester side for subsequent attestations. On the other hand, if the attestation fails, there are several possible attestation results including any combination of the following cases: $C D_{i}$ integrity success/fail, RProcess $s_{i}$ un/authenticated, and Policy $_{i}$ success/fail. To assist the attestee reconfiguration, the attester also sends a representation of the policy violation graph to the attestee. Moreover, with this policy violation graph, the attester ranks the violation graph and measures the trustworthiness of the attestee, which is explained in Section 6.

\section{Graph-Based Policy Analysis}

As we have discussed, existing attestation solutions such as TCG and IMA lack the expressiveness of the attestation result. Instead of a boolean value for an attestation result, DR@FT adopts a graph-based policy analysis mechanism, where a policy violation graph can be constructed for identifying all policy violations on the attestee side. We further introduce a risk model based on a ranking scheme, which implies how severe the discovered policy violations are and how efficiently they can be resolved. This section illustrates the graph-based integrity analysis and violation identification. We explain the risk model in next section.

\subsection{Information Flow and Policy Graph}

For information flow purpose, all operations between subjects and objects in a policy can be classified as write_like or read_like [15] and operations between subjects can be expressed as calls. Depending on the types of operations, information flow relationships can be identified. If a subject $x$ can write an object $y$, there is information flow from $x$ to $y$, which is denoted as write $(x, y)$. On the other hand, if a subject $x$ can read an object $y$, there is information flow from $y$ to $x$ denoted as $\operatorname{read}(y, x)$. Another situation is that if a subject $x$ can call another subject $y$, there is information flow from $y$ to $x$, which is denoted as $\operatorname{call}(y, x)$.

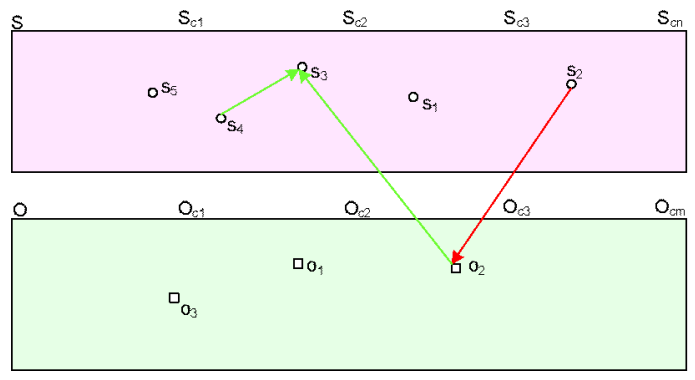

Fig. 2. Policy graph: The link between $s_{2}$ and $o_{2}$ represents write operation, $s_{3}$ and $o_{2}$ for read operation, and $s_{4}$ and $s_{3}$ for call operation.

Moreover, the information flow relationships between subjects and objects can be further described through flow transitions. In a policy, if a subject $s_{1}$ can write an object $o$ which can be read by another subject $s_{2}$, it implies that there is an information flow transition from subject $s_{1}$ to subject $s_{2}$, denoted as flowtrans $\left(s_{1}, s_{2}\right)$. Also, if a subject $s_{2}$ can call a subject $s_{1}$, there is a flow transition from $s_{1}$ to $s_{2}$. A sequence of flow transitions between two subjects represents an information flow path.

With information flow and flow transitions between subjects and objects, we define a policy graph as follows:

Definition 3. A Policy Graph of a system is a directed graph $G=(V, E)$, where the set of vertices $V$ represents all subjects and objects in the system, and the set of edges $E=V \times V$ represents all information flow relations between subjects and objects. That is,

- $\quad V \subseteq V_{o} \cup V_{s}$, where $V_{o}$ and $V_{s}$ are the sets of nodes that represent objects and subjects, respectively;

- $E \subseteq E_{r} \bigcup E_{w} \bigcup E_{c}$. Given the vertices $v_{s 1}, v_{s 2} \in V_{s}$ separately representing subjects $s 1$ and $s 2$, and vertices $v_{o} \in V_{o}$ representing object $o,\left(v_{s 1}, v_{o}\right) \in$ $E_{w}$ if and only if write $(s 1, o),\left(v_{o}, v_{s 2}\right) \in E_{r}$ if and only if $\operatorname{read}(o, s 2)$, and $\left(v_{s 1}, v_{s 2}\right) \in E_{c}$ if and only if $\operatorname{call}(s 1, s 2)$.

We use semantic substrates to display policies [25]. We divide a canvas into different areas based on the classification of entities (subjects and objects) and then layout nodes expressing the entities into corresponding areas. We also use nonspacial cues (e.g., color or shape) to emphasize certain nodes or a group of nodes. Fig. 2 shows some example policy rules within a policy graph. The $Y$-axis is divided into regions, where each region contains nodes representing entities such as subjects and objects. Furthermore, in each region, nodes representing entities of different classifications are placed in different spaces along with the $X$-axis. For subjects and objects in a policy, $S_{c 1} \ldots S_{c n}$ and $O_{c 1} \ldots O_{c m}$ separately represent certain classifications. Different colors and shapes are used to distinguish the identification of different nodes. Circles and rectangles are used to represent subjects and objects, respectively. Relationships between subjects and objects are expressed with lines in different colors or shapes. For instance, the write operation between subject $s_{2}$ and object $o_{2}$ is expressed with a red link.

\subsection{Policy Violation Graph}

An information flow represents how data flow among system subjects. A sequence of information flow transitions 


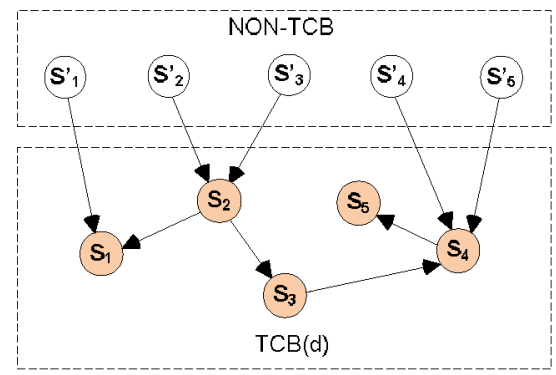

(a) A policy violation graph

\begin{tabular}{|c|l|}
\hline Subject & SubjectRank \\
\hline $\mathrm{S}_{1}$ & 0.2799 \\
$\mathrm{~S}_{2}$ & 0.3999 \\
$\mathrm{~S}_{3}$ & 0.0799 \\
$\mathrm{~S}_{4}$ & 0.4319 \\
$\mathrm{~S}_{5}$ & 0.3456 \\
\hline
\end{tabular}

(b) SubjectRank in $\mathrm{TCB}(\mathrm{d})$

\begin{tabular}{|c|l|}
\hline Path & PathRank \\
\hline$\left\langle\mathrm{S}_{1}^{\prime}, \mathrm{S}_{1}\right\rangle$ & 0.2799 \\
$\left\langle\mathrm{~S}_{2}^{\prime}, \mathrm{S}_{2}\right\rangle$ & 0.8104 \\
$\left\langle\mathrm{~S}^{\prime}{ }_{3}, \mathrm{~S}_{2}\right\rangle$ & 0.8104 \\
$\left\langle\mathrm{~S}^{\prime}{ }_{4}, \mathrm{~S}_{4}\right\rangle$ & 0.6048 \\
$\left\langle\mathrm{~S}_{5}^{\prime}, \mathrm{S}_{4}\right\rangle$ & 0.6048 \\
\hline
\end{tabular}

(c) PathRank between NON-TCB and $\mathrm{TCB}(\mathrm{d})$

Fig. 3. Example policy violation graph and rank. SubjectRank and PathRank indicate severity of violating paths.

between two subjects shows an information flow path. Applying our domain-based isolation approach, policy violations can be detected to identify information flows from low-integrity subjects to high-integrity subjects. Corresponding information flow paths representing such policy violations are named violation paths.

Based on domain-based integrity model, we can discover two kinds of violation paths, direct violation paths and indirect violation paths. A direct violation path is a one-hop path through which an information flow can go from a lowintegrity subject to a high-integrity subject. We observe that information flows are transitive in general. Therefore, there may exist information flows from a low-integrity subject to a high-integrity subject via several other subjects. This multihop path is called indirect violation path. All direct and indirect violation paths belonging to a domain can construct a policy violation graph for this domain.

Definition 4. A policy violation graph for a domain $d$ is a directed graph $G^{v}=\left(V^{v}, E^{v}\right)$ :

- $V^{v} \subseteq V_{N T C B}^{v} \cup V_{T C B d}^{v} \cup V_{T C B s}^{v}$ where $V_{N T C B}^{v}, V_{T C B d}^{v}$ and $V_{T C B s}^{v}$ are subject vertices containing in direct or indirect violation paths of domain $d$ and belong to NON-TCB $T C B_{d}$, and $T C B_{s}$, respectively.

- $\quad E^{v} \subseteq E_{N d}^{v} \cup E_{d T}^{v} \cup E_{N T}^{v} \cup E_{N T C B}^{v} \cup E_{T C B d}^{v} \cup E_{T C B s}^{v}$ where

$$
\begin{aligned}
& E_{N d}^{v} \subseteq\left(V_{N T C B}^{v} \times V_{T C B d}^{v}\right), \\
& E_{d T}^{v} \subseteq\left(V_{T C B d}^{v} \times V_{T C B s}^{v}\right), \\
& E_{N T}^{v} \subseteq\left(V_{N T C B}^{v} \times V_{T C B s}^{v}\right), \\
& E_{N T C B}^{v} \subseteq\left(V_{N T C B}^{v} \times V_{N T C B}^{v}\right), \\
& E_{T C B d}^{v} \subseteq\left(V_{T C B d}^{v} \times V_{T C B d}^{v}\right),
\end{aligned}
$$

and $E_{T C B s}^{v} \subseteq\left(V_{T C B s}^{v} \times V_{T C B s}^{v}\right)$, and all edges in $E^{v}$ are contained in direct or indirect violation paths of domain $d$.

Fig. 3a shows an example of policy violation graph which examines information flows between NON-TCB and $T C B_{d}{ }^{2}$ Five direct violation paths are identified in this graph: $<S_{1}^{\prime}, S_{1}>,<S_{2}^{\prime}, S_{2}>,<S_{3}^{\prime}, S_{2}>,<S_{4}^{\prime}, S_{4}>$, and $<S_{5}^{\prime}, S_{4}>$ across all the boundaries between NON-TCB and $T C B_{d}$. Also, eight indirect violation paths exist. For example, $\left\langle S_{2}^{\prime}, S_{5}\right\rangle$ is a four-hop violation path passing through other three $T C B_{d}$ subjects $S_{2}, S_{3}$, and $S_{4}$.

2. Similarly, the information flows between NON-TCB and $T C B_{s}$, and between $T C B_{d}$ and $T C B_{s}$ can be examined accordingly.
Algorithm 2 realizes the processing to identify policy violation graph with inputs of a policy graph and updated policy file. First, a function policyParse parses the updated policy information into subjects, objects, and their relationships with permission mapping. Second, we load this information onto the previously generated Policy graph with functions changeNodes and changeLinks, respectively. Then, it determines if there is a new information flow generated on this graph with a function getnewFlow. Third, we need to check if this new information flow violates our domain-based isolation rules using a function identifyViolation. Through this approach, rather than analyzing all the policy rules and all information flows for each attestation, we verify the new policy through only checking the updated policy rules and the newly identified information flows. The complexity of this policy analysis algorithm is $O(n n+n l+n t)$, where $n n$ represents the number of changed subjects and objects, $n l$ is the number of changed subject and object relationships in the policy update file; and $n t$ represents the number of changed TCB in the TSL file.

Algorithm 2. Finding Integrity Violations with Policy Graph and Updates

Input: Initial trusted policy graph $G_{0}$, policy updating file $P_{u}$, TSL file $T_{\text {listi }}$, policy explanation file $F_{e}$, permission mapping File $F_{p}$, subject classification file $F_{s}$, object classification file $F_{o}$

Output: Policy violation graph $G_{\text {flow }}$

Policy $_{u}:=$ policyParse $\left(P_{u}, F e, F_{p}, F_{s}, F_{o}\right)$

$N_{\text {list }}:=$ get UpdateSO $\left(\right.$ Policy $\left._{u}\right)$

foreach $r_{i} \in R_{\text {list }}$ do changeNodes $\left(n_{a}, G_{0}\right)$

$L_{\text {list }}:=$ getUpdateLink $\left(\right.$ Policy $\left._{u}\right)$

foreach $l_{a} \in L_{\text {list }}$ do changeLinks $\left(l_{a}, G_{0}\right)$ getnewFlow $\left(l_{a}, G_{0}\right)$

foreach $t_{a} \in T_{\text {list }}$ do changeTCB $\left(t_{a}, G_{0}\right)$ $G_{\text {flow }}:=$ identifyViolation $\left(t_{a}, G_{0}\right)$

\section{Ranking Policy Violation Graph}

In order to explore more features of policy violation graphs and facilitate efficient policy violation detection and resolution, we introduce a scheme for ranking policy violation 
graphs. There are two steps to rank a policy violation graph. First, $T C B_{d}$ subjects in the policy violation graph are ranked based on dependency relationships among them. The rank of a $T C B_{d}$ subject shows reachable probability of lowintegrity information flows from NON-TCB subjects to the $T C B_{d}$ subject. In addition, direct violation paths in the policy violation graph are evaluated based on the ranks of $T C B_{d}$ subjects to indicate the severity of these paths which allow low-integrity information to reach $T C B_{d}$ subjects. The ranked policy violation graphs are valuable for system administrators as they need to estimate the risk level of a system and provide a guide for choosing appropriate strategies for resolving policy violations efficiently.

\subsection{Ranking Subjects in $\mathrm{TCB}_{d}$}

Our notation of SubjectRank (SR) in policy violation graphs is a criterion that indicates the likelihood of low-integrity information flows coming to a $T C B_{d}$ subject from NONTCB subjects through direct or indirect violation paths. The ranking scheme we adopt is a similar process of rank analysis applied in hyper-text link analysis system, such as Google's PageRank [26] that utilizes a link structure provided by hyperlinks between web pages to gauge their importance. Comparing with PageRank which focuses on analyzing a web graph, where the entries are any web pages contained in the web graph, the entries of low-integrity information flows to $T C B_{d}$ subjects in a policy violation graph are only identified NON-TCB subjects.

Consider a policy violation graph with $N$ NON-TCB subjects, and $s_{i}$ is a $T C B_{d}$ subject. Let $N\left(s_{i}\right)$ be the number of NON-TCB subjects from which low-integrity information flows could come to $s_{i}, N^{\prime}\left(s_{i}\right)$ the number of NON-TCB subjects from which low integrity information flows could directly reach to $s_{i}, \operatorname{In}\left(s_{i}\right)$ a set of $T C B_{d}$ subjects pointing to $s_{i}$, and $\operatorname{Out}\left(s_{j}\right)$ a set of $T C B_{d}$ subjects pointed from $s_{j}$. The probability of low-integrity information flows reaching a subject $s_{i}$ is given by

$$
S R\left(s_{i}\right)=\frac{N\left(s_{i}\right)}{N}\left(\frac{N^{\prime}\left(s_{i}\right)}{N\left(s_{i}\right)}+\left(1-\frac{N^{\prime}\left(s_{i}\right)}{N\left(s_{i}\right)}\right) \sum_{s_{j} \in \operatorname{In}\left(s_{i}\right)} \frac{S R\left(s_{j}\right)}{\left|\operatorname{Out}\left(s_{j}\right)\right|}\right) .
$$

SubjectRank can be interpreted as a Markov Process, where the states are $T C B_{d}$ subjects, and the transitions are the links between $T C B_{d}$ subjects which are all evenly probable. While a low-integrity information flow attempts to reach a highintegrity subject, it should select an entrance (a NON-TCB subject) which has the path(s) to this subject. Thus, the possibility of selecting correct entries to a target subject is $\frac{N\left(s_{i}\right)}{N}$. After selecting correct entries, there still exist two ways, through direct violation or indirect violation paths, to reach a target subject. Therefore, the probability of flow transition from a subject is divided into two parts: $\frac{N^{\prime}\left(s_{i}\right)}{N\left(s_{i}\right)}$ for direct violation paths and $1-\frac{N^{\prime}\left(s_{i}\right)}{N\left(s_{i}\right)}$ for indirect violation paths. The $1-\frac{N^{\prime}\left(s_{i}\right)}{N\left(s_{i}\right)}$ mass is divided equally among the subject's successors $s_{j}$, and $\frac{S R\left(s_{j}\right)}{\left|\operatorname{lut}_{j}\left(s_{j}\right)\right|}$ is the rank value derived from $s_{j}$.

Fig. $3 b$ displays a result of applying (1) to the policy violation graph shown in Fig. 3a. Note that even though a subject $s_{4}$ has two direct paths from NON-TCB subjects like a subject $s_{2}$, the rank value of $s_{4}$ is higher than the rank value of $s_{2}$, because there is another indirect flow path to $s_{4}$ (via $s_{3}$ ).

\subsection{Ranking Direct Violation Path}

We further define PathRank (PR) as the rank of a direct violation path, ${ }^{3}$ which reflects the severity of the violation path through which low-integrity information flows may come to $T C B_{d}$ subjects. Direct violation paths are regarded as the entries of low integrity data to $T C B_{d}$ in policy violation graph. Therefore, the ranks of direct violation paths give a guide for system administrators to adopt suitable defense countermeasures for solving identified violations. To calculate PathRank accurately, three conditions are needed to be taken into account: 1 ) the number of $T C B_{d}$ that low-integrity flows can reach through this direct violation path; 2) SubjectRank of reached $T C B_{d}$ subjects; and 3 ) the number of hops to reach a $T C B_{d}$ subject via this direct violation path.

Suppose $<s_{i}^{\prime}, s_{j}>$ is a direct violation path from a NONTCB subject $s_{i}^{\prime}$ to a $T C B_{d}$ subject $s_{j}$ in a policy violation graph. Let Reach $\left(\left\langle s_{i}^{\prime}, s_{j}\right\rangle\right)$ be a function returning a set of $T C B_{d}$ subjects to which low-integrity information flows may go through a direct violation path $\left\langle s_{i}^{\prime}, s_{j}\right\rangle, S R\left(s_{l}\right)$ the rank of a $T C B_{d}$ subject $s_{l}$, and $H_{s}\left(s_{i}^{\prime}, s_{l}\right)$ a function returning the hops of the shortest path from a NON-TCB subject $s_{i}^{\prime}$ to a $T C B_{d}$ subject $s_{l}$. The following equation is utilized to compute a rank value of the direct violation path $\left\langle s_{i}^{\prime}, s_{j}\right\rangle$.

$$
P R\left(<s_{i}^{\prime}, s_{j}>\right)=\sum_{\left.s_{l} \in \operatorname{Reach}\left(<s_{i}^{\prime}, s_{j}\right\rangle\right)} \frac{S R\left(s_{l}\right)}{H_{s}\left(s_{i}^{\prime}, s_{l}\right)} .
$$

Fig. 3c shows the PathRank of the example policy violation graph, which is calculated by the above-defined equation. For example, $<s_{2}^{\prime}, s_{2}>$ has a higher rank than $\left\langle s_{1}^{\prime}, s_{1}\right\rangle$, because $\left\langle s_{2}^{\prime}, s_{2}\right\rangle$ may result in low-integrity information flows to reach more or important $T C B_{d}$ subjects than $<s_{1}^{\prime}, s_{1}>$.

\subsection{Evaluating Trustworthiness}

Let $P_{d}$ be a set of all direct violation paths in a policy violation graph. The entire rank, which can be considered as a risk level of the system, can be computed as follows:

$$
\text { RiskLevel }=\sum_{<s_{i}^{\prime}, s_{j}>\in P_{d}} P R\left(<s_{i}^{\prime}, s_{j}>\right) .
$$

The calculated risk level could reflect the trustworthiness of an attestee. Generally, the lower risk level indicates the higher trustworthiness of a system. When an attestation is successful and there is no violation path being identified, the risk level of the attested system is zero, which means an attestee has the highest trustworthiness. On the other hand, when an attestation is failed, corresponding risk level of a target system is computed. A selective service could be achieved based on this fine-grained attestation result. That is, the number of services provided by a service provider to the target system may be decided with respect to the trust level of the target system. Hence, a system administrator could refer to this attestation result as the evaluation of her/ his system as well as resolution guidelines since this quantitative response would give her a proper justification to adopt countermeasures for improving the trustworthiness of a target system.

3. It is possible that a system administrator may also want to evaluate indirect violation paths for violation resolution. In that case, our ranking scheme could be adopted to rank indirect violation paths as well. 


\subsection{Attestee Reconfiguration}

When the current system state of an attestee does not satisfy integrity requirements, a system administrator may need to change the system configuration based on the attestation result to enhance the system integrity and improve its trust level. The total rank of all violation paths provides a measurement of risk level of a system and thus reflects the system's trustworthiness. A system administrator is able to adopt different countermeasures to resolve identified violations with respect to the different rank values of violation paths. We summarize several options as follows:

- Changing $C D_{i}$ and $T S L_{i}$ : in case an attestation is failed because of loading malicious or unknown codes or data into the system, removing these codes and data can change the system state to be trusted, while there is no change to the policy.

- Identifying filter: if a filter is identified along with the information flow path that causes a violation, the filter can be added to $C D_{i}$ and $T S L_{i}$. In this case, the violation is treated as a false alarm and there is no change to the policy graph.

- Changing Policy P $_{i}$ we are able to modify policy for violation resolution, either by excluding subjects or objects from the violated information flow paths, or by replacing subjects or objects with more restricted privileges. Based on the violated information flow paths and corresponding ranks, a system administrator can first eliminate highly ranked violation paths since they generally involve more violations and more $T C B_{d}$ subjects. Through modifying policy rules, $P$ flow $_{i}$ is changed to satisfy our domain isolation requirements. In other words, an attestee can maintain integrity requirements by modifying system policies properly for a newly installed software which interacts with TSL subjects.

- Adding filter: we can also introduce a new filter subject that acts as a gateway along a violation flow path between NON-TCB and TCB subjects. By including such a filter in $C D_{i}$ and $T S L_{i}$, the violation can be resolved in subsequent attestations.

\section{IMPLEMENTATION AND EVALUATION}

We have implemented DR@FT to evaluate its effectiveness and measure the performance. This section first describes our experimentation setup, followed by implementation details, effectiveness evaluation, and performance study.

Our attestee platform is a Lenovo ThinkPad X61 with Intel Core 2Duo Processor L7500 1.6 GHz, 2 GB RAM, and Atmel TPM. We enable SELinux with the default policy based on the current distribution of SELinux [22]. To measure the attestee system with TPM, we update the Linux kernel to 2.6.26.rc8 with the latest IMA implementation [23], where SELinux hooks and IMA functions are enabled.

Having IMA enabled, we configure the measurement of the attestee information. After the attestee system kernel is booted, we mount the sysfs file system and inspect the measurement list values in ascii_runtime_measurements and ascii_bios_measurements. Fig. 5a shows a sample of the measurement list.

\subsection{Attestation Implementation}

We start from a legitimate attestee and make measurements of the attestee system for the later verification. To invoke a new attestation request from the attester, the attestation reporting daemon runs in the attestee and monitors the attestee system. This daemon is composed of two main threads: one monitors and obtains new system state measurements, and the other monitors and obtains the policy updates of the attestee. The daemon is also measured and the result can be obtained through the legitimate attestee. Thus, the integrity of the daemon can be verified later by the attester. In case the attestee system state is updated due to new software installation, changing policy, and so on, the corresponding thread of the daemon automatically obtains the new measurement values as discussed in Section 4. The daemon then securely transfers the attestation information to the attester based on available security mechanisms supported by the trusted authority.

After receiving the updated system information from the attestee, the measurement module of the attester checks the received measurements against the stored PCR to prove its integrity. To analyze the revised attestee policy, the policy analysis module is developed as a daemon, which is derived from a policy analysis engine [27]. We extend the engine to support information flow query functions, so as to identify violated information flows from the updated policy rules based on domain-based isolation rules. We use JDK1.6 and other necessary Java libraries to develop the main analysis components. We implemented graph drawing with graph package Piccolo [28]. We also accommodate the attestation procedures presented in Section 4.2, as well as our rank scheme to evaluate the trustworthiness of the attestee.

\subsection{Identifying TCBs and Resolving Violations}

To evaluate the proposed attestation framework, we attest our testbed platform with Apache web server installed. To configure the trusted subject list of the Apache domain, we first identify $T C B_{s}$ based on the reference monitor-based TCB identification [21], including the integrity measurement, monitoring agents, and daemon. For $T C B_{d}$ of the Apache, we identify the Apache information domain, Apache $T C B_{d}$, including httpd_t and httpd_suexec_t, and the initial filters sshd_t, passwd_t, su_t, through the domain-based isolation principles. Both $T C B_{s}$ and $T C B_{d}$ are initially identified with a graphical policy analysis tool [27], and then all possible policy violations are detected as well according to our domain-based integrity model. Fig. 4a shows an example with automatically identified policy violations, which indicates write_like operations from NON-TCB subjects to objects that can be read by $T C B_{d}$ subjects. We then apply the system reconfiguration strategies discussed in Section 6.4 to resolve these violations.

Update TCB. We observe that some violations are generated due to the reason that all information flows to some subjects in turn flows to TCB subjects. We then include these subjects in the TCB. For example, in our evaluation policy, httpd_awstats_script_t can flow to $T C B_{d}$ subjects through httpd_awstats_script_rw_t. At the same time, it is flown in by many NON-TCB subjects through some common types such as devtty_t. Hence, we ignore the violations caused by this awstats_script and 


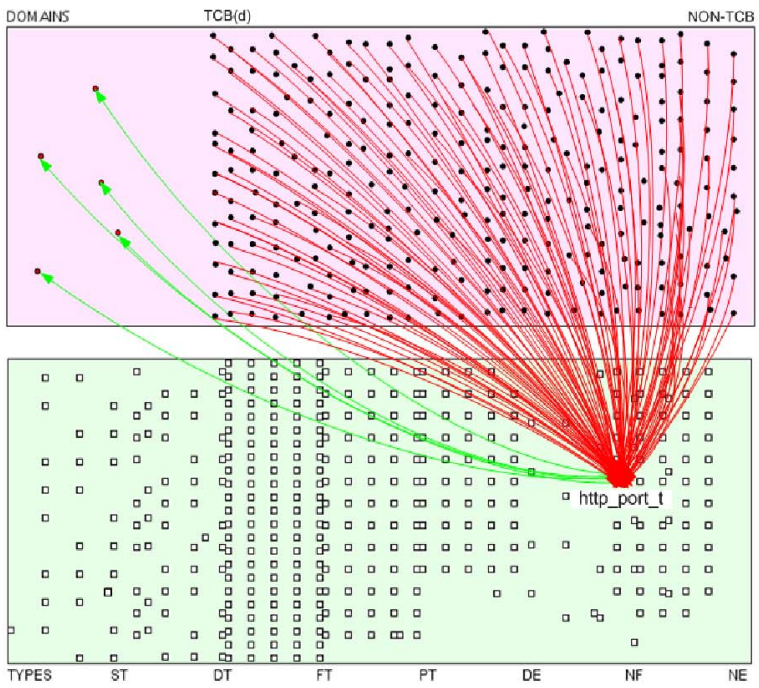

(a). Policy violations caused by NON-TCB subjects flow to $T C B(d)$ subjects through httpd_port_t

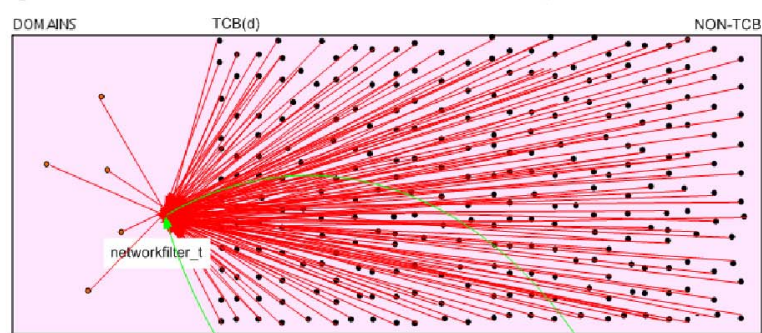

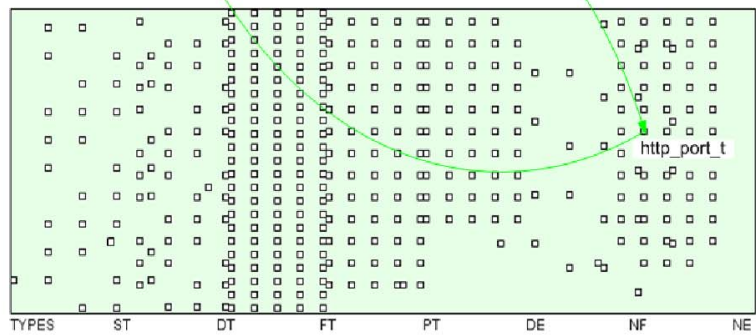

(b). Policy violation caused by http_port_t is solved through adding networkfilter into the related policies.

Fig. 4. Policy violations and corresponding resolution for Apache domain.

include it into $T C B_{d}$. Similar situation occurs for httpd_apcupsd_cgi_script_t and httpd_prewikka_script_t. However, httpd_staff_script_t cannot be included into $T C B_{d}$ since it would lead unlimited file access for the staff services such as staff_t, staff_ mozilla_t, and staff_mplayer_t.

Remove Policy Rules. Another way for resolving policy violations is to remove related policy statements. For example, webalizer_t is to label a tool for analyzing the $\log$ files of web server and is not necessary to modify web server information. To resolve the policy violations caused by webalizer_t due to the write access to httpd_sys_content_t, we remove the policy rule stating write_like operation between webalizer_t and httpd_sys_content_t.

Modify Policy Rules. Many policy violations are caused because related subjects or objects are given too much privileges. Hence, rather than just removing related policy statements, we also need to replace these subjects or objects
TABLE 1 Identified System TCB and Apache TCB

\begin{tabular}{|l|l|l|}
\hline \multicolumn{3}{|c|}{ System TCB } \\
\hline kernel_t & load_policy_t & initrc_t \\
\hline mount_t & ipsec_mgmt_t & useradd_t \\
\hline hwclock_t & admin_passwd_exec_t & cardmgr_t \\
\hline kudzu_t & sshd_login_t & restorecon_t \\
\hline syslogd_t & sysadm_t & getty_t \\
\hline dpkg_t & logrotate_t & snmpd_t \\
\hline bootloader_t & quota_t & init_t \\
\hline ldconfig_t & apt_t & sshd_t \\
\hline passwd_t & newrole_t & klogd_t \\
\hline automount_t & checkpolicy_t & fsadm_t \\
\hline lvm_t & local_login_t & setfiles_t \\
\hline ima_t & rpdaemon_t & \\
\hline \multicolumn{2}{|l|}{ Apache domain TCB } \\
\hline httpd_suexec_t & httpd_awstats_script_t & httpd_t \\
\hline httpd_helper_t & httpd_php_t & httpd_rotatelogs_t \\
\hline httpd_sysadm_script_t & httpd_prewikka_script_t & httpd_apcupsd_cgi_script_t
\end{tabular}

with more restricted rights. For example, for policy violations caused by read and write accesses to initrc_devpts_t, our solution is to redefine initrc_devpts_t by introducing initrc_devpts_t, system_initrc_devpts_t, and *_daemon_initrc_devpts_t. ${ }^{4}$ Corresponding policy rules are modified as follows:

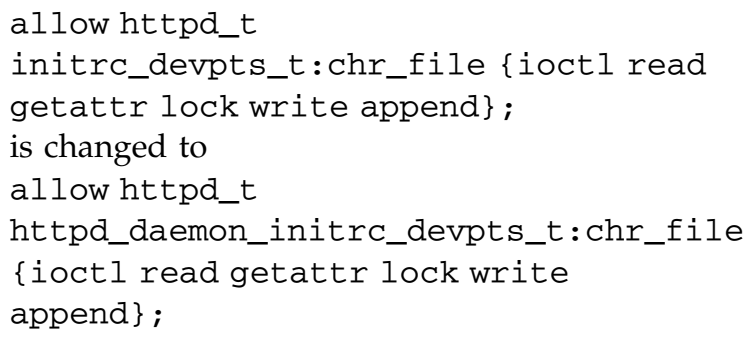

Add Filter. Based on our domain-based integrity model, a filter can be introduced into policies to resolve policy violations. For example, to remove the violations caused by http_port_t, we introduce a network filter subject as follows:

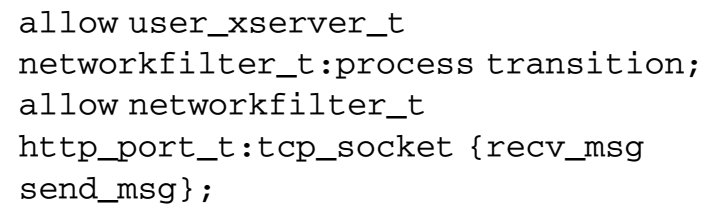

After the modification is applied, the original policy violations are eliminated. In general, to validate the result of a policy modification, we need to recheck the relationships between the policy violation related domains and types. Comparing Figs. $4 \mathrm{a}$ with $4 \mathrm{~b}$, we can observe that all read operations between $T C B_{d}$ and type http_port_t are removed. Also, the write operations between NON-TCB and http_port_t are also removed. Instead, a new domain networkfilter_t is added, which has write and read operations on http_port_t. Also, all $T C B_{d}$ and NON-TCB subjects can transit to this new domain type.

The initial $T C B_{d}$ is then adjusted during the process of resolving violations. Table 1 shows the final system TCB and Apache TCB identified with our approach. We then install unverified codes and data to evaluate the effectiveness of our attestation framework.

4. *Representing the corresponding service name. 


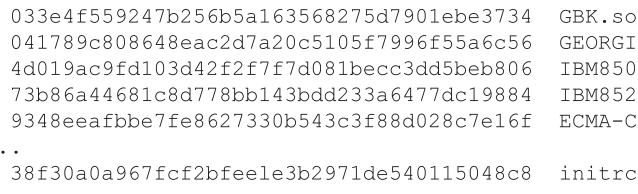

(a) Measurement example

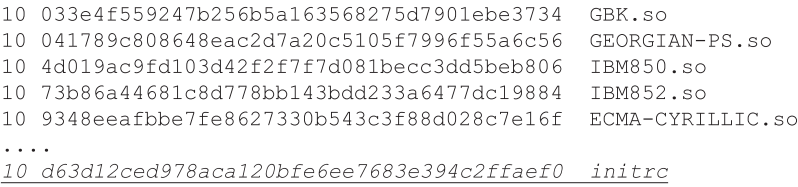

(b) Measurement after installing rootkit

Fig. 5. Measurement list example consisting a PCR location, file SHA-1 hash, and file name.

\subsection{Installing Malicious Code}

We first install a Linux rootkit, which gains administrative control without being detected. Here, we assign the rootkit with the domain unconfined_t, which enables information flows to domain initrc_t which labels initrc process in the $T C B_{s}$ of the attestee.

Following the framework proposed in Section 4, the attestee system is measured from the bootstrap with the configured IMA. After getting the new measurement values, the reporting daemon sends these measurements to the attester. Note that there is no policy update in this experiment. Different from IMA, we only measure the $T C B_{s}$ and $T C B_{d}$ subjects. After getting the measurements from the attestee, the attester verifies them by analyzing the measured hash values. Fig. 5 shows partial measurements of a trusted initial system state and the changed state because of the installed rootkit. The difference between these two measurements indicates that the original initrc is altered, thus the attester confirms that the attestee is not in a trusted state.

\subsection{Identifying Vulnerable Software}

In this experimentation, we install a vulnerable software called Mplayer on the attestee side. Mplayer is a media player and encoder software which is susceptible to several integer overflows in the real video stream dumuxing code. These flaws allow an attacker to cause a denial of service or potentially execution of the arbitrary code by supplying a deliberately crafted video file. During the installation of a Mplayer, a Mplayer policy module is also loaded into the attestee policy. In this policy module, there are several different subjects such as usr_mplayer_t, staff_mplayer_t, and sysadm_mplayer_t. Also, some objects are defined in security policies such as user_mplayer_home_t, staff_mplayer_home_t, and sysadm_mplayer_home_t. After the Mplayer is installed, the attestation daemon finds that the new measurement of Mplayer is generated and the security policy of the system is changed. As the Mplayer does not belong to $T C B_{s}$ and Apache $T C B_{d}$, the attestation daemon does not need to send the measurements to the attester. Consequently, the daemon only computes the security policy updates and sends the information to the attester.

Upon receiving the updated policies, we analyze these updates and obtain a policy violation graph as shown in Fig. 6. Through objects such as cifs_t, sysadm_devtps_t, and ncsd_var_run_t, information flows from Mplayer can reach Apache domain. In addition, rank values are calculated and shown in the policy violation graph, which guides effective violation resolutions. For example, there are three higher ranked paths including paths from sysadm devpts_t to httpd_t, from ncsd_var_run_t to httpd_rotatelogs_t, and from cifs_t to httpd_ prewikka_script_t. Meanwhile, a risk level value (1.2584) reflecting the trustworthiness of the attestee system is computed based on the ranked policy violation graph.
Once receiving the attestation result shown in Fig. 6, the system administrator can resolve the violation that has the higher rank than others. Thus, the administrator can first resolve the violation related to httpd_t through introducing httpd_sysadm_devpts_t.

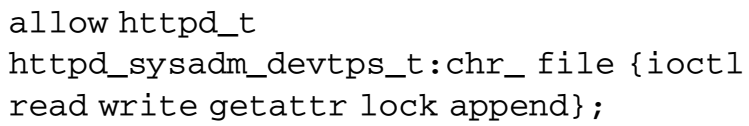

After the policy violation resolution, the risk level of the attestee system is lowered to 0.7315 . Continuously, after the attestee resolves all the identified policy violations and the risk level is decreased to be zero, the attestation daemon gets a new policy update and sends it to the attester. Upon receiving this update, the attester verifies whether the identified information flows violate domain-based isolation integrity rules since these flows are within the NON-TCB even though there are new information flows compared to the stored Policy. Thus, an attestation result is generated which indicates the risk level (in this case, zero) of the current attestee system. Consequently, a new trusted system state is built for the attestee. In addition, the information of this new trusted system state is stored in the attester side for the later attestation.

\subsection{Performance}

To examine the scalability and efficiency of DR@FT, we investigate how well the attestee measurement agent, attestation daemon, and the attester policy analysis module scale along with increased complexity, and how efficiently DR@FT performs by comparing it with traditional approaches.

In DR@FT, the important factors influencing the attestation performance include system updates and policy changes. Hence, we evaluate the performance of DR@FT by changing codes and data to be measured and modifying the security policies. Based on our study, we observe that normal policy increased or decreased no more than $40 \mathrm{~KB}$ when installing or uninstalling software. Also, a system

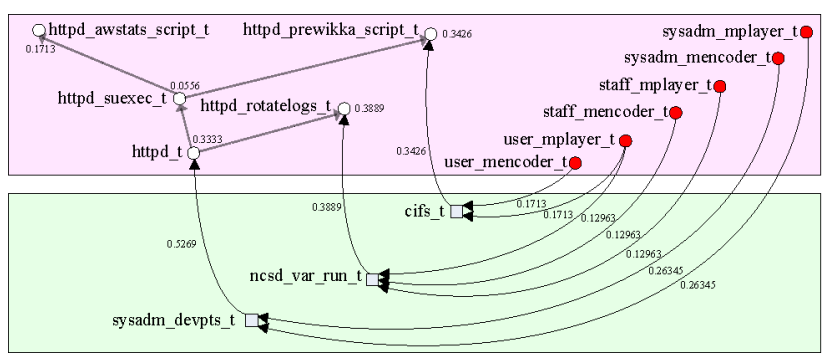

Fig. 6. Information flow verification for Mplayer. The links show the information flow from Mplayer (filled circle nodes) to Apache (unfilled nodes). The rank values on the paths indicate the severity of violations. 
TABLE 2

Attestation Performance Analysis (in Seconds)

\begin{tabular}{|l|l|l|l|l|l|l|l|}
\hline Policy Change & \multicolumn{3}{|c|}{ Dynamic } & \multicolumn{3}{c|}{ Static } \\
\hline Size & $T_{\text {Pupdate }}$ & $T_{\text {send }}$ & $T_{\text {Panalysis }}$ & Overhead & $T_{P \text { send }}$ & $T_{\text {Panalysis }}$ & Overhead \\
\hline 0 & 0.23 & 0 & 0 & 0.23 & 14.76 & 90.13 & 104.89 \\
\hline$-0.002 \mathrm{MB}$ (Reduction) & 0.12 & 0.002 & 0.02 & 0.14 & 14.76 & 90.11 & 104.87 \\
\hline$-0.019 \mathrm{MB}$ (Reduction) & 0.08 & 0.01 & 0.03 & 0.12 & 14.74 & 89.97 & 104.34 \\
\hline$-0.024 \mathrm{MB}$ (Reduction) & 0.04 & 0.02 & 0.03 & 0.09 & 14.74 & 89.89 & 104.23 \\
\hline $0.012 \mathrm{MB}$ (Addition) & 0.37 & 0.01 & 0.03 & 0.41 & 14.77 & 90.19 & 104.96 \\
\hline $0.026 \mathrm{MB}$ (Addition) & 0.58 & 0.02 & 0.03 & 0.63 & 14.78 & 90.33 & 105.11 \\
\hline $0.038 \mathrm{MB}$ (Addition) & 0.67 & 0.03 & 0.04 & 0.74 & 14.79 & 90.46 & 105.25 \\
\hline
\end{tabular}

administrator does not make the enormous changes over the policy. Therefore, the performance is measured with the range from zero to around $40 \mathrm{~KB}$ in terms of policy size.

Performance on the attestee side. Based on DR@FT, the attestee has three main factors influencing the attestation performance. 1) Time spent for the measurement: based on our experimentation, the measurement time increases roughly linearly with the size of the target files. For example, measuring policy files with 17.2 and $20.3 \mathrm{MB}$ requires 14.63 and 18.26 seconds, respectively. Measuring codes around $27 \mathrm{MB}$ requires 25.3 seconds. 2) Time spent for identifying policy updates $T_{\text {Pupdate }}$ : based on the specification in Section 4, policy updates are required to be identified and sent to the attester. As shown in Table 2, for a system policy which is with the size of $17.2 \mathrm{MB}$ at its precedent state, the increase of the policy size requires more time for updating the policy and vice versa. 3) Time spent for sending policy updates $T_{\text {Psend }}$ : basically, the more policy updates, the higher overhead was observed.

Performance on the attester side. In DR@FT, the measurement verification is relatively straightforward. At the attester side, the time spent for policy analysis $T_{\text {Panalysis }}$ mainly influences its performance. As shown in Table 2, the analysis time roughly increases when the policy change rate increases.

Comparison of dynamic and static attestation. To further evaluate the efficiency of DR@FT, we compare the overhead of DR@FT with a static attestation. In the static approach, the attestee sends all system state information to an attester, and the attester verifies the entire information step by step. As shown in Table 2, the time spent for the static attestation is composed of $T_{\text {Psend }}$ and $T_{\text {Panalysis }}$, which represent the time for sending policy module and analyzing them, respectively. Obviously, the dynamic approach can dramatically reduce the overhead compared to the static approach. It shows that DR@FT is an efficient way when policies on an attestee are updated frequently.

\section{Conclusion}

We have presented a dynamic remote attestation framework called DR@FT for efficiently verifying if a system satisfies integrity protection property and indicates integrity violations which determine its trustworthiness level. The integrity property of our work is based on an information flow-based domain isolation model, which is utilized to describe the integrity requirements and identify integrity violations of a system. To achieve the efficiency and effectiveness of remote attestation, DR@FT focuses on system changes on the attestee side. We have extended our intuitive policy analysis engine to represent integrity violations with a rank scheme. In addition, our results show that our dynamic approach can dramatically reduce the overhead compared to the static approach. We believe such a comprehensive method would help system administrators reconfigure the system with more efficient and strategic manner.

There are several directions that our attestation framework can be extended to. First, DR@FT attests system configurations according to security policies, while it does not attest the trustworthiness of dynamic contents of a system such as runtime state of applications. Second, our risk evaluation does not explain the tolerable risk level and relevant system properties. In addition, all verification tasks are performed at the attester side. The attester may need to delegate some attestation tasks to trusted components at the attestee or other trusted side. Our future work would seek a more flexible and systematic way to address these issues.

\section{ACKNOWLEDGMENTS}

The work of Gail-Joon Ahn and Hongxin Hu was partially supported by the grants from the US National Science Foundation (NSF-IIS-0900970 and NSF-CNS-0831360) and the US Department of Energy (DE-SC0004308). The work of Gail-Joon Ahn and Wenjuan Xu was also partially supported by the grants from the US National Science Foundation (NSF-IIS-0242393) and the US Department of Energy Early Career Principal Investigator Award (DE-FG02-03ER25565).

\section{REFEREnCES}

[1] "Trusted Computing Group," https:/ / www. trustedcomputinggroup.org, 2011.

[2] R. Sailer, X. Zhang, T. Jaeger, and L. van Doorn, "Design and Implementation of a TCG-Based Integrity Measurement Architecture," Proc. 13th Conf. USENIX Security (SSYM '04), 2004.

[3] L. Chen, R. Landfermann, H. Löhr, M. Rohe, A.-R. Sadeghi, and C. Stüble, "A Protocol for Property-Based Attestation," Proc. First ACM Workshop Scalable Trusted Computing (STC '06), 2006.

[4] V. Haldar, D. Chandra, and M. Franz, "Semantic Remote Attestation: A Virtual Machine Directed Approach to Trusted Computing," Proc. Third Conf. Virtual Machine Research and Technology Symp. (VM '04), 2004.

[5] T. Jaeger, R. Sailer, and U. Shankar, "PRIMA: Policy-reduced Integrity Measurement Architecture," Proc. 11th ACM Symp. Access Control Models and Technologies (SACMAT '06), 2006.

[6] K.J. Biba, "Integrity Consideration for Secure Compuer System," Technical Report 3153, Mitre Corp., 1977.

[7] T. Fraser, "Lomac: Low Water-Mark Integrity Protection for Cots Environment," Proc. IEEE Symp. Security and Privacy (SP '00), May 2000.

[8] R.S. Sandhu, "Lattice-Based Access Control Models," Computer, vol. 26, no. 11, pp. 9-19, Nov. 1993.

[9] U. Shankar, T. Jaeger, and R. Sailer, "Toward Automated Information-Flow Integrity Verification for Security-Critical Applications," Proc. Network and Distributed System Security Symp. (NDSS), 2006. 
[10] T. Jaeger, R. Sailer, and X. Zhang, "Analyzing Integrity Protection in the Selinux Example Policy," Proc. 12th Conf. USENIX Security Symp. (SSYM '03), 2003.

[11] U. Shankar, T. Jaeger, and R. Sailer, "Toward Automated Information-Flow Integrity Verification for Security-Critical Applications," Proc. Network and Distributed System Security Symp. (NDSS), The Internet Soc., http://dblp.uni-trier.de/db/conf/ ndss/ndss2006.html\#ShankarJS06, 2006.

[12] S. Smalley, "Configuring the Selinux Policy," http://www.nsa. gov/SELinux/docs.html, 2003.

[13] B. Hicks, S. Rueda, L.S. Clair, T. Jaeger, and P. McDaniel, "A Logical Specification and Analysis for Selinux mls Policy," ACM Trans. Information Systems Security, vol. 13, no. 3, pp. 1-31, 2010.

[14] Tresys Technology APOL, http://www.tresys.com/selinux/, 2011.

[15] J. Guttman, A. Herzog, and J. Ramsdell, "Information Flow in Operating Systems: Eager Formal Methods," Proc. Workshop Issues in the Theory of Security (WITS), 2003.

[16] B. Sarna-Starosta and S.D. Stoller, "Policy Analysis for SecurityEnhanced Linux," Proc. Workshop Issues in the Theory of Security (WITS), pp. 1-12, Apr. 2004.

[17] W. Xu, M. Shehab, and G. Ahn, "Visualization Based Policy Analysis: Case Study in Selinux," Proc. ACM Symp. Access Control Models and Technologies, 2008.

[18] G. Ahn, W. Xu, and X. Zhang, "Systematic Policy Analysis for High-Assurance Services in Selinux," Proc. IEEE Workshop Policies for Distributed Systems and Networks, pp. 3-10, 2008

[19] M. Alam, X. Zhang, M. Nauman, T. Ali, and J.-P. Seifert, "ModelBased Behavioral Attestation," Proc. 13th ACM Symp. Access Control Models and Technologies (SACMAT '08), 2008.

[20] Trusted Computer System Evaluation Criteria. United States Govt. Dept. of Defense (DOD), Profile Books, 1985.

[21] A.P. Anderson, "Computer Security Technology Planning Study," Technical Report ESD-TR-73-51, vol. II, 1972.

[22] S. Smalley, "Configuring the Selinux Policy," http://www.nsa. gov/SELinux/docs.html, 2003.

[23] "LIM Patch," http://lkml.org/lkml/2008/6/27, 2011.

[24] N. Provos, M. Friedl, and P. Honeyman, "Preventing Privilege Escalation," Proc. 12th Conf. USENIX Security Symp. (SSYM '03), p. 11, Aug. 2003.

[25] M. Green, "Toward a Perceptual Science of Multidimensional Data Visualization: Bertin and Beyond," http://www.ergogero. com/dataviz/dviz2.html, 1998.

[26] S. Brin and L. Page, "The Anatomy of a Large-Scale Hypertextual Web Search Engine," Computer Networks and ISDN Systems, vol. 30, nos. 1-7, pp. 107-117, 1998.

[27] W. Xu, X. Zhang, and G.-J. Ahn, "Towards System Integrity Protection with Graph-Based Policy Analysis," Proc. 23rd Ann. IFIP WG 11.3 Working Conf. Data and Applications Security, 2009.

[28] "Piccolo ToolKit," http://www.cs.umd.edu/hcil/jazz/. 2011.

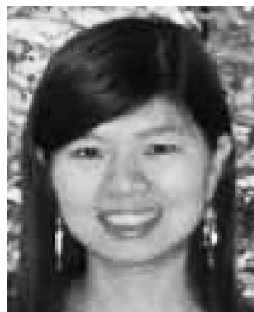

Wenjuan $\mathrm{Xu}$ received the $\mathrm{PhD}$ degree in information technology from the University of North Carolina at Charlotte. She is an assistant professor of the Department of Computer Science and Information Technologies at Frostburg State University, Maryland. Her main research interest include access control and information security.

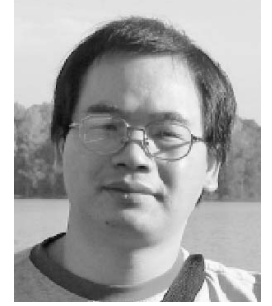

Xinwen Zhang received the $\mathrm{PhD}$ degree in information security from George Mason University, Fairfax in 2006. He is a research staff member at Huawei Research Center at Santa Clara. His research interests include security policies, models, architectures, and mechanism in general computing and networking systems. His recent research focuses on secure and trusted network infrastructure, cloud computing, and mobile platforms and systems. $\mathrm{He}$ is a member of the IEEE.

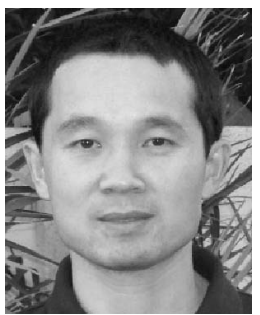

Hongxin $\mathrm{Hu}$ is currently working toward the $\mathrm{PhD}$ degree from the School of Computing, Informatics, and Decision Systems Engineering, Ira A. Fulton School of Engineering, Arizona State University, Tempe. He is also a member of the Security Engineering for Future Computing Laboratory, Arizona State University. His current research interests include access control models and mechanisms, security and privacy in social networks, security in distributed and cloud computing, network and system security, and secure software engineering. He is a student member of the IEEE.

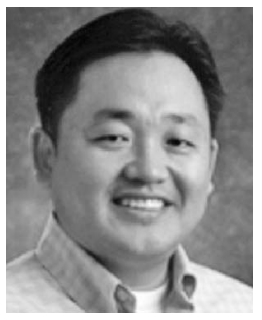

Gail-Joon Ahn received the PhD degree in information technology from George Mason University, Fairfax, Virginia, in 2000. He is an associate professor in the School of Computing Informatics, and Decision Systems Engineering, Ira A. Fulton Schools of Engineering and the Director of Security Engineering for Future Computing Laboratory, Arizona State University. His research interests include information and systems security, vulnerability and risk management, access control, and security architecture for distributed systems, which has been supported by the US National Science Foundation, National Security Agency, US Department of Defense, US Department of Energy, Bank of America, Hewlett Packard, Microsoft, and Robert Wood Johnson Foundation. He is a recipient of the US Department of Energy CAREER Award and the Educator of the Year Award from the Federal Information Systems Security Educators Association. He was an associate professor at the College of Computing and Informatics, and the Founding Director of the Center for Digital Identity and Cyber Defense Research and Laboratory of Information Integration, Security, and Privacy, University of North Carolina, Charlotte. He is a senior member of the IEEE.

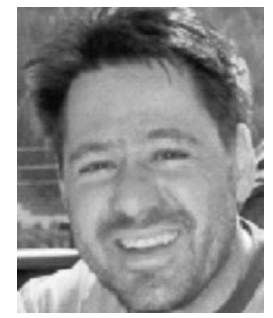

Jean-Pierre Seifert received the PhD degree in the year 2000 with Professor Dr. Claus Schnorr one of the most important theoretician in the field of secure information systems from JohannWolfgang-Goethe-University at Frankfurt/Main where he studied computer science and mathematics. Afterward, he gained intensive practical experience working in the research and development departments for hardware Security at Infineon, Munich and Intel. At Intel, (2004-2006), he has been responsible for the design and integration of new CPU security instructions for micro processors that are going to be integrated in all Intel micro processors. From 2007-2008, he developed for Samsung Electronics the worldwide first commercial secure cell phone based on the Linux operating system. Since the end of 2008, he has been a professor heading the group of security in Telecommunications at TU Berlin and Deutsche Telekom Laboratories. In 2002, he has been honored by Infineon with the award inventor of the Year and has received as well two Intel Achievement Awards in 2005 for his new CPU security instructions for the Intel micro processors. Approximately 40 patents have been granted to him in the field of computer security. He is a member of the IEEE.

For more information on this or any other computing topic, please visit our Digital Library at www.computer.org/publications/dlib. 